\title{
Cell Compartment Stabilization in the Prebiotic Environment
}

\author{
Chrissa Karagiannis $^{1 *}$, David Deamer ${ }^{1}$
}

Amphiphilic self-assembly is integral to membrane formation in the cellular environment. Prebiotic structures are guided by carbonaceous meteorites representative of components found on primitive Earth, such as simpler amphiphiles and fatty acids. The membrane assembly is an essential step to the protection of protocells in the hydrothermal environment, where they are introduced to high temperatures and osmotic tensions in hydrative and dehydrative cycles. Stabilization factors allow vesicles to compartmentalize from the larger hydrothermal environment, preventing the denaturation of non-template directed nucleic acid chains and other synthesized peptides. The presence of protein homopolymers in carbonaceous materials and their synthesis in simulated prebiotic conditions make them likely candidates for stabilizing lipid vesicles. In this study, protein homopolymers were analyzed for efficiency in decreasing vesicle permeability through kinetic spectrophotometry and retention of fluorescent labels in internal lamellar layers. We looked closely at the homopolymer glycine in conjunction with phosphatidylglycerol as a stabilizing agent due to its abundance in carbonaceous meteorites, the formation of polymers in hydrothermal conditions, and achiral nature. We then investigated the formation of pores with amphiphilic tripeptides in preventing lysis of vesicles from osmotic stress. We show that homopolymers produce vesicular structures dependent on side chains and discuss the significance of osmotic stress and charge residue on the structure of vesicles, permeability, and the prebiotic environment. We did not find significant protective effects from homopolymers or simple pores but discuss the importance of stabilization in reducing the sensitivity of cells to the outer environment. Protective effects are essential to the proliferation and development of protocells into their modern counterparts.

\section{INTRODUCTION}

Membranous enclosures are important to the development of life because they maintain systems of interacting molecules within a semi-permeable compartment. The permeable barriers of biological membranes today are composed of phospholipid bilayers with two fatty acid chains esterified to a glycerol phosphate. Because phospholipids are the product of complex metabolic processes catalyzed by enzymes, it is unlikely that they were available on prebiotic Earth, so there must have been an abiotic source of lipid-like amphiphiles (Shimoyama, Naraoka, Komiya, \& Harada, 1989). Carbonaceous meteorites serve as a guide to the kinds of organic compounds likely to be present. This suite of organics includes monomers such as amino acids, nucleobases, and monocarboxylic acid meteorites. Some of the monocarboxylic acids have been shown to form membranous vesicles (Apel, Deamer, \& Mautner, 2002; Namani \& Deamer, 2008), which become more stable if present as a mixture with a monoglyceride or alcohol. Specialized

\footnotetext{
${ }^{1}$ Department of Chemistry and Biochemistry, University of California-Santa Cruz, Physical Sciences, Room 405B, 1156 High Street, Santa Cruz CA 95060
}

*To whom correspondence should be addressed: ce.karagiannis@yahoo.com

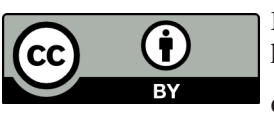

Except where otherwise noted, this work is licensed under https://creativecommons.org/licenses/by/4.0

doi:10.22186/jyi.35.1.29-37 cytoskeletal proteins such as spectrin in red blood cell membranes further stabilize biological membranes (Cramer, Engelman, Von Heijne, \& Rees, 1992).

Stabilizing a primitive membrane is an essential first step for the self-assembly of protocells in a hydrothermal environment on the early Earth. It has been suggested that one of the first stabilizing factors in a primitive membrane would be interactions between a peptide polymer and a simplified lipid bilayer (Damer \& Deamer, 2015). Each protocell is presumed to have a membranous boundary that provides an environment in which polymerization and combinatorial chemistry can occur. Without a stabilizing factor, fragile membranes can be disrupted by environmental stresses and release their contents, preventing continued synthesis and survival of nucleic acid polymers. This research is the first of its kind to tackle stabilization factors of membranes in the hydrothermal prebiotic environment.

Glycine is the most abundant amino acid present in carbonaceous meteorites and was also one of the first products identified by Miller (1953) in his experiments using gas mixtures exposed to electrical discharge. Glycine is achiral with only two hydrogens on the alpha carbon and can exist in both hydrophobic and hydrophilic environments. Glycine monomers are typically incorporated in the amino acid sequences of proteins at points requiring high conformational flexibility (Oliver \& Deamer, 1994). Glycine has also been demonstrated to form polymers in hydrothermal conditions (Fujioka et al., 2009).

In searching for the equivalent of a prebiotic cytoskeletal polymer, we hypothesize that the conformational flexibility and 
hydrogen bonding capacity of polyglycine makes it a likely candidate to stabilize prebiotic membranes and decrease the permeability of vesicles in highly osmotic environments. In the experiments reported here, we investigated vesicles prepared from phosphatidylglycerol as a model of a relatively fragile but temperature insensitive prebiotic membrane. Using microscopy, we monitor the effects of positively, negatively, and neutrally charged homopolymers on simple vesicles and analyze their permeability using kinetic spectrophotometry. We then focus on polyglycine as a promising stabilization factor and use internal fluorescence labeling to confirm the presence of homopolymer glycine bound lipid vesicles and determine homopolymer glycine's effectiveness in protecting vesicles from osmotic stress. This study confirms the capacity for simple homopolymers to bind to vesicle surfaces, brings insight into the importance of protection from environmental stressors and progresses the search for a successful stabilization factor in the prebiotic environment.

\section{MATERIALS AND METHODS}

\section{Permeability Experiments of Charged and Uncharged Poly- peptide Residues}

Microscopy of polylysine, polyarginine, polyglutamate, and polyglycine

All polyamino acids used in the following experiments were acquired from Sigma-Aldrich. We made twelve solutions of polylysine (MW 150,000), polyarginine (MW 41,400), polyglutamate (MW 80,000), and polyglycine (MW 85,943.5) in 1:1, 10:1, and 100:1 solutions of phosphatidylglycerol $(5 \mathrm{mg} / \mathrm{mL})$ to respective polypeptide $(1 \mathrm{mg} / \mathrm{mL})$ and suspended them in $0.2 \mathrm{M}$ glycerol. We checked $\mathrm{pH}$ and we added Tris Buffer (.1M, pH 10) to bring the solutions over $\mathrm{pH} 7$ and ensure protonation of the lysine and arginine side chains, and deprotonation of the glutamate side chain. We extruded half of each solution in a 1-micron filter, giving twelve unfiltered and twelve filtered solutions of $1: 1,10: 1$, and 100:1 phosphatidylglycerol to polypeptide ratios. We carried out spectrophotometry experiments and dried them down, rehydrated with $30 \mu \mathrm{l} \mathrm{H} 2 \mathrm{O}$, and visualized the filtered solutions with phase microscopy at 400X magnification using a Zeiss Axiovert $200 \mathrm{mi}-$ croscope. We then compared these solutions to a phosphatidylglycerol control.

Spectrophotometry of polylysine, polyarginine, polyglutamate, and polyglycine

We carried out kinetic spectrophotometry experiments at 500nm absorption for 300 seconds at 5-second intervals. For each experiment, we placed $100 \mu 1$ of the phosphatidylglycerol-polypeptide solutions in $\mathrm{mL} 2 \mathrm{M}$ glycerol at 50 seconds. We conducted additional control of a phosphatidyl glycine only solution to ensure a comparison of permeability. We first carried out the experiments with unfiltered solutions, then with solutions extruded through the 1 -micron filter. $30 \mu 1$ of the unfiltered solutions were dried down, resuspended in $30 \mu \mathrm{l}$ water, and visualized under a microscope.

The permeability of phosphatidyl glycerol with L-valglycylglycine
To determine the permeability of phosphatidylglycerol in the presence of a potentially pore-forming tripeptide, we made $1: 1,5: 1$, and 10:1 weight ratios of phosphatidylglycerol to L-valglycylglycine (VGG) and suspended them in $\mathrm{H} 2 \mathrm{O}$, sonicated them for three minutes, dried them down on a slide, resuspended them and visualized them with phase microscopy. We also sonicated, dried down, and visualized a phosphatidylglycerol only solution via phase microscopy for comparison. We made a separate $10 \mathrm{mg} / \mathrm{mL}$ solution of phosphatidylglycerol in $0.2 \mathrm{M}$ glycerol and made a $1: 1$ by weight ratio solution of the phosphatidylglycerol to the tri-peptide which was extruded through a 1-micron filter. We conducted the first experiment at 500nm for 180 seconds, with 5-second cyclic intervals; the second test was done at $500 \mathrm{~nm}$ for 600 seconds, with 30 -second cyclic intervals. For the first experiment, we placed $100 \mu 1$ of the phosphatidylglycerol and VGG solution in $1 \mathrm{~mL} 2 \mathrm{M}$ glycerol solution at 60 seconds and graphed the results. For the second test, we placed $100 \mu \mathrm{l}$ of the $0.2 \mathrm{M}$ glycerol solution at 100 seconds and graphed the results. Each test included a control solution of only phosphatidylglycerol in $0.2 \mathrm{M}$ glycerol.

Standard curve of polyglycine

We diluted a solution of $500 \mu \mathrm{g} / \mathrm{mL}$ of polyglycine to $10,20,30$, 40,50 , and $100 \mu \mathrm{g} / \mathrm{mL}$ solutions and each solution was checked on a Nanodrop for the absorbance three times. A standard curve was made using average absorption data from each solution at $200 \mathrm{~nm}$. made using average absorption data from each solution at $200 \mathrm{~nm}$.

Measuring the absorbance of polyglycine and estimating the ratio of bound polyglycine to phosphatidyl glycerol

To determine a quantitative estimate of individual glycine molecules bound to the inner and outer layers of phosphatidylglycerol, we made solutions of $5: 1,10: 1$, and $20: 1$ phosphatidylglycerol to polyglycine in $\mathrm{H} 2 \mathrm{O}$. The solutions were centrifuged for 10 minutes at $14 \mathrm{~K}$. We discarded the supernatants and resuspended the pellets in $100 \mu \mathrm{l} \mathrm{H} 2 \mathrm{O}$. We then added $150 \mu \mathrm{l}$ butanol and $50 \mu \mathrm{l} \mathrm{H} 2 \mathrm{O}$ to the solutions and discarded the top layer to separate the phosphatidylglycerol vesicles from bound polyglycine. We then measured the solutions on a Nanodrop. The absorbance values were compared with the standard curve of polyglycine at 500nm, and we made estimates of the percent of polyglycine bound to phosphatidylglycerol based on the graph. We also made mathematical estimates of the ratio of bound glycine to phosphatidylglycerol with the values taken from the Nanodrop.

Calculating the number of polyglycine residues attached to phosphatidyl glycerol surface

The ratio of lipid to bound polyglycine was calculated for 5:1, 10:1 and 20:1 solutions of phosphatidylglycerol to polyglycine.

\section{Polyglycine labeling with fluorescamine}

We completed fluorescence labeling to confirm the binding of polyglycine to the lipid vesicles through Phase Microscopy. We added $0.75 \mathrm{~mL}$ of Sodium Borate buffer to $0.75 \mathrm{~mL}$ polyglycine $(5 \mathrm{mg} / \mathrm{mL}) .0 .5 \mathrm{~mL}$ fluorescamine $(30 \mathrm{mg} / 100 \mathrm{~mL})$ to the solution as the solution was vortexed. The solution was spun in $4.5 \mathrm{~mL}$ centrifugal filters (Microcon YM-10) at $14 \mathrm{~K}$ RPM for 20 minutes. 

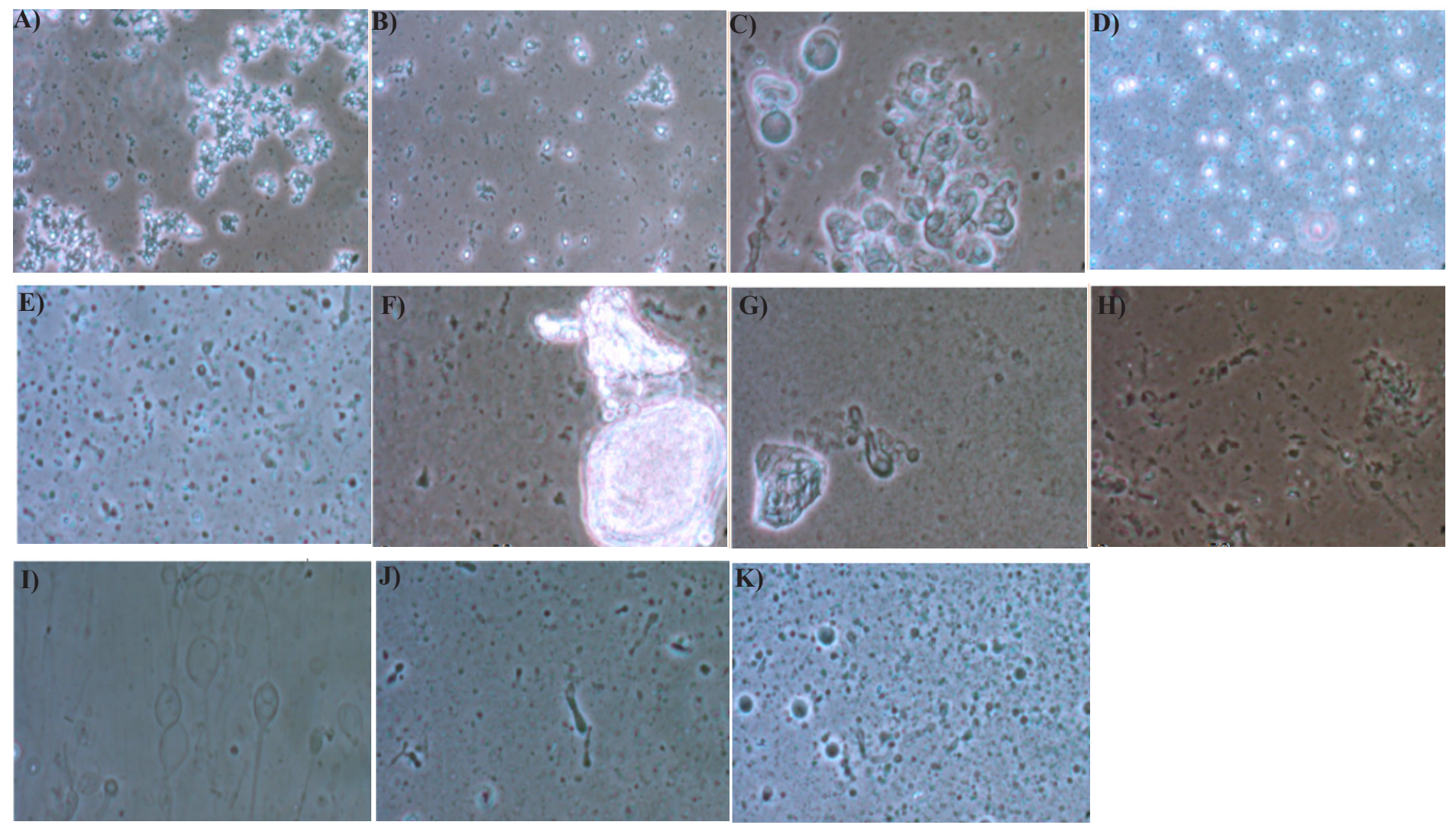

Figure 1. Microscopy images of filtered polylysine, polyglycine, polyarginine, and polyglutamate. A) 1:1 phosphatidyl glycerol to polylysine with a 1-micron filter. B) 10:1 phosphatidyl glycerol to polylysine filtered with a 1-micron filter. C) 100:1 phosphatidyl glycerol to polylysine filtered with a 1-micron filter. D) 10:1 phosphatidyl glycerol to polyarginine filtered with a 1-micron filter. E) 100:1 phosphatidyl glycerol to polyarginine filtered with a 1-micron filter. F) 1:1 phosphatidyl glycerol to polyglutamate filtered with a 1-micron filter. G) 10:1 phosphatidyl glycerol to polyglutamate fil-tered with a 1-micron filter. H) 100:1 phosphatidyl glycerol to polyglutamate filtered with a 1-micron filter. I) 1:1 phosphatidyl glycerol to polyglycine filtered with a 1-micron filter. J) 10:1 phosphatidyl glycerol to polyglycine filtered with a 1-micron filter. K) 100:1 phosphatidyl glycerol to polyglycine filtered with a 1-micron filter.

We discarded the supernatant, opened the top filter and added $50 \mu 1$ $\mathrm{H} 2 \mathrm{O}$ to each solution, then spun each solution twice at $1 \mathrm{~K}$ RPM for three minutes. We then added $20 \mu 1$ phosphatidylglycerol to $20 \mu 1$ of the labeled polyglycine and dried down the solution on a microscope slide, rehydrated it with $\mathrm{H} 2 \mathrm{O}$ and visualized it using UV trans-illuminated phase microscopy.

Measuring the Osmotic Effects of Liposomes using Fluorescence

We created two fluorescence solutions to determine whether there were general osmotic effects on the liposomes during dehydration and rehydration or fluorescence specific osmotic effects. We made fluorescamine-labeled polyglycine using the steps outlined above. We made the second fluorescent solution with phosphatidylglycerol $(10 \mathrm{mg} / \mathrm{mL})$ and Pyranine. We added $1 \mathrm{~mL}$ Pyranine to two $1 \mathrm{~mL}$ phosphatidylglycerol solutions and extruded the solutions through a 1-micron filter. We placed the solutions through a column to remove excess pyranine and then dried down the solutions and resuspended each in either $30 \mu \mathrm{l} \mathrm{H} 2 \mathrm{O}, 30 \mu 1 \mathrm{mM}$ sucrose, or $30 \mu \mathrm{l}$ $100 \mathrm{mM}$ sucrose to prevent the fusion of vesicles upon dehydration.
We dried down the solutions and resuspended them as follows: 5:1 polyglycine to labeled phosphatidylglycerol resuspended in $\mathrm{H} 2 \mathrm{O}$; 10:1 polyglycine to labeled phosphatidylglycerol resuspended in $\mathrm{H} 2 \mathrm{O} ; 5: 1$ polyglycine to unlabeled phosphatidylglycerol resuspended in $\mathrm{H} 2 \mathrm{O}$; 10:1 polyglycine to unlabeled phosphatidylglycerol resuspended in $\mathrm{H} 2 \mathrm{O}$; labeled phosphatidylglycerol with $1 \mathrm{mM}$ sucrose resuspension; labeled phosphatidylglycerol with $100 \mathrm{mM}$ sucrose resuspension; unlabeled phosphatidylglycerol with $1 \mathrm{mM}$ sucrose resuspension; and unlabeled phosphatidylglycerol with $100 \mathrm{mM}$ sucrose resuspension.

Determining Lysis Prevention using polyglycine and polyglutamate

We made a $5 \mathrm{~mL}$ solution of phosphatidylglycerol $(10 \mathrm{mg} / \mathrm{mL})$ and sonicated it for 5 minutes. After sonication, we mixed five solutions with $0.5 \mathrm{~mL}$ pyranine, $0.5 \mathrm{~mL}$ phosphatidylglycerol and added polypeptide solutions as follows; $0.5 \mathrm{~mL}$ pyranine with $0.5 \mathrm{~mL}$ phosphatidylglycerol only; $1: 1$ phosphatidylglycerol to polyglycine $(0.5 \mathrm{mg} / \mathrm{mL})$ solution; $1: 1$ phosphatidylglycerol to polyglycine $(2 \mathrm{mg} / \mathrm{mL})$ solution; $1: 1$ phosphatidylglycerol to poly- 
A)

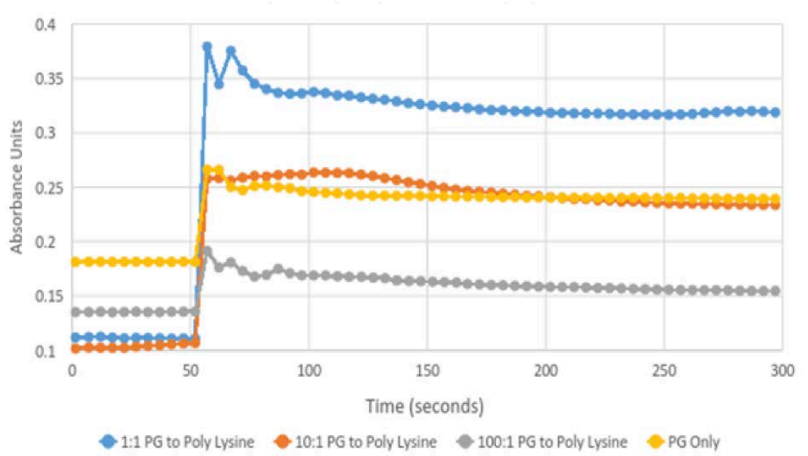

C)

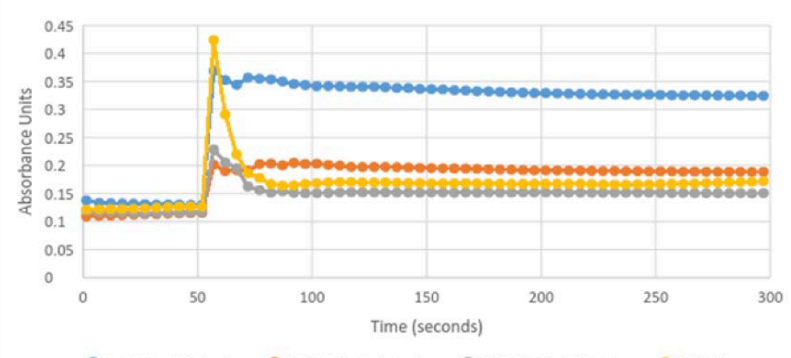

E)

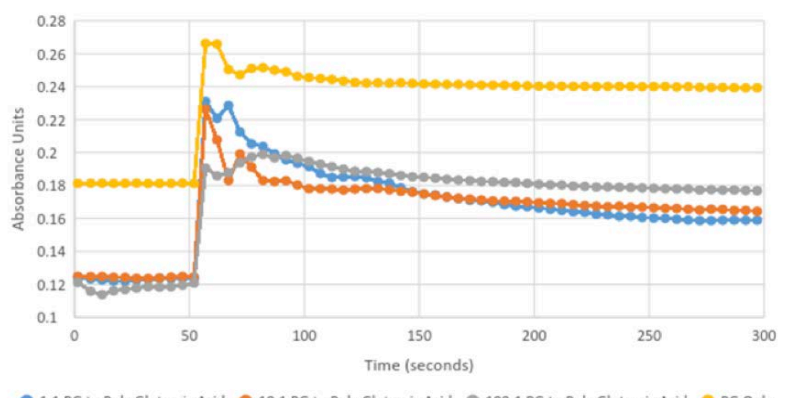

G)

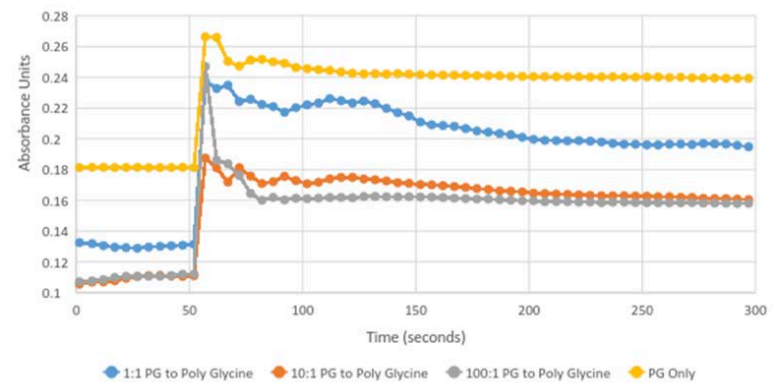

B)

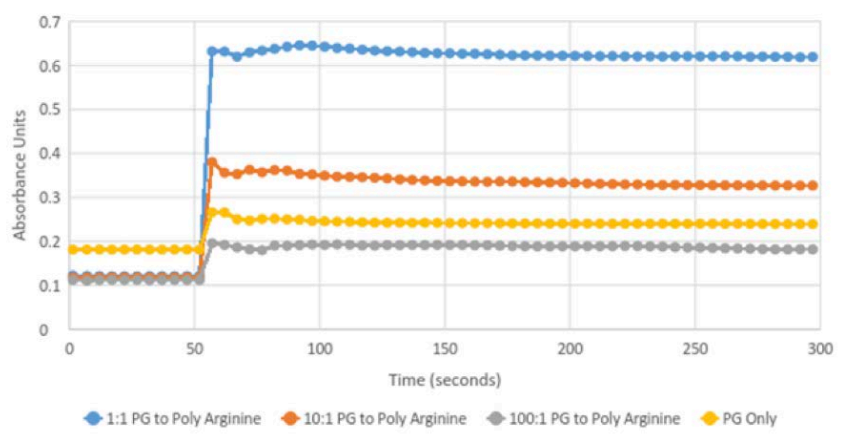

D)

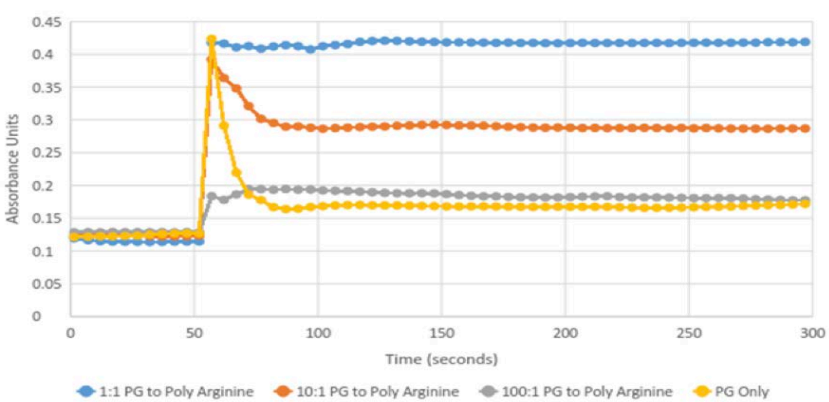

F)

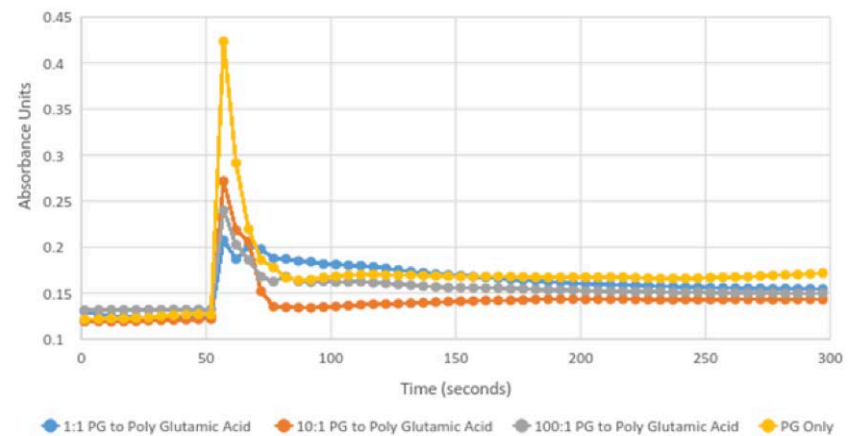

H)

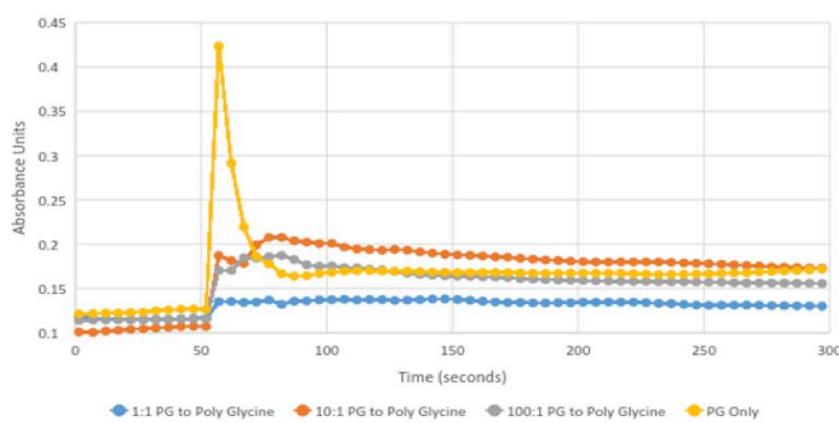

Figure 2. Spectrophotometry of polylysine, polyarginine, polyglutamate, and polyglycine. A) Permeability changes of unfiltered 1:1, 10:1, and 100:1 phosphatidyl glycerol to polylysine. B) Permeability changes of filtered 1:1, 10:1, and 100:1 phosphatidyl glycerol to polylysine. C) Permeability changes of unfiltered 1:1, 10:1, and 100:1 phosphatidyl glycerol to polyarginine. D) Permeability changes of filtered 1:1, 10:1, and 100:1 phosphatidyl glycerol to polyarginine. E) Permeability changes of unfiltered 1:1, 10:1, and 100:1 phosphatidyl glycerol to polyglutamate. F) Permeability changes of filtered 1:1,10:1, and 100:1 phosphatidyl glycerol to polyglutamate. G) Permeability changes of unfiltered 1:1, 10:1 and 100:1 phosphatidylglycerol to polyglycine. H) Permeability changes of filtered 1:1, 10:1, and 100:1 phosphatidyl glycerol to polyglycine. 

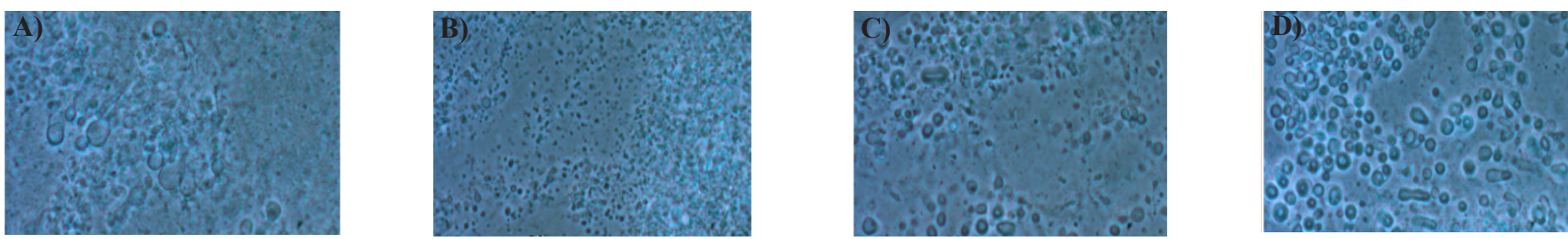

Figure 3. Permeability of phosphatidyl glycerol with L-valglycylglycine. A) 1:1 phosphatidyl glycerol to L-valglycylglycine in $\mathrm{H}_{2} \mathrm{O}$. B) $5: 1$ phosphatidyl glycerol to L-valglycylglycine in $\mathrm{H}_{2} \mathrm{O}$. C) 10:1 phosphatidyl glycerol to L-valglycylglycine in $\mathrm{H}_{2} \mathrm{O}$. D) Phosphatidyl glycerol in $\mathrm{H}_{2} \mathrm{O}$.

glutamate $(0.5 \mathrm{mg} / \mathrm{mL})$ solution; and a $1: 1$ phosphatidylglycerol to polyglutamate $(2 \mathrm{mg} / \mathrm{mL})$ solution. We dried down the solutions on a slide and rehydrated them with water, then visualized by phase and fluorescence microscopy. We placed polyglutamate $(2 \mathrm{mg} / \mathrm{mL})$ in a solution with $0.5 \mathrm{~mL}$ phosphatidyl glycerol, sonicated it for five minutes, dried it down on a slide, rehydrated it with $\mathrm{H} 2 \mathrm{O}$, and then visualized it with phase and fluorescence microscopy.

\section{RESULTS}

All scales are in microns, unless otherwise noted.

Microscopy of Filtered polylysine, polyglycine, polyarginine, and polyglutamate

The addition of 1:1 and 10:1 ratios of positively charged polyarginine and polylysine produced inhibitive effects on the liposomes, preventing formation of vesicles and aggregation clusters of positively charged homopolymers with phosphatidyl glycerol. As the lipid to peptide ratios reached diluted concentrations, the aggregative effects dissipated, indicating that the inhibitory effects were a result of the large presence of positively charged side chains in solution. Large aggregates of protein and were observed in the 1:1 phosphatidylglycerol to polyglutamate solution. However, the interaction between negatively charged polyglutamate and phosphatidylglycerol did not completely inhibit vesicle formation, as compared to positively charged homopolymers. Aggregated liposomes took on a shrunken, minimal form, but did not form large clusters as with the positively charged peptides. Aggregative and inhibitive effects dissipated with the increased dilution of phosphatidylglycerol to polyglutamate ratios. Polyglycine was the only peptide whose inclusion in the lipid solution did not produce inhibitive effects on the vesicles despite the presence of excess clusters of polyglycine in the 1:1 solution. The polyglycine liposomes contained large, multilamellar vesicles, microtubule shaped vesicles, and an excess of small vesicles. The 1:1 solution was the only polyglycine solution which contained an excess number of vesicles smaller than 1-micron, unable to be captured in photos but visualized in the microscope. Other polyglycine solutions contained vesicles of varying sizes, ranging from large, multilamellar vesicles, to tiny, unilamellar vesicles. The results can be visualized in Figure 1, Panels A-K.

Spectrophotometry of polylysine, polyarginine, polyglutamate, and polyglycine

All filtered solutions were placed through a 1-micron extruder to create uniformity in size among the vesicles. Although the filtered and unfiltered solutions vary slightly in absorption units, the area underneath the peaks of the experimental data shows little change in permeability with the addition of polylysine to phosphatidylglycerol solutions. Results for the filtered and unfiltered polylysine to phosphatidylglycerol are visualized in Figure 2, Panels A and B.

We were unable to observe a change in permeability between the phosphatidylglycerol solution and solutions containing polyarginine. The 1:1 polyarginine solution showed the largest amount of aggregates in the phase microscopy, and the area under the peak of the $1: 1,10: 1$, and 100:1 solutions showed little change in permeability from the phosphatidylglycerol only solution. Both polylysine solutions and the polyarginine solutions showed similar absorption values. Polyarginine solutions are visualized in Figure 2, Panel C and D.

While spectrophotometry units differed between the filtered and unfiltered solutions, permeability results remained unchanged. There was no difference in the area under the curve for the phosphatidylglycerol only solutions from the polyglutamate solutions, visualized in Figure 2, Panel E and F. There was a significant change in absorption units in the polyglutamate experiments back to values close to the original absorption units of glycerol prior to the addition of liposomes and protein. The difference in the baseline absorption values 50 seconds after addition of the liposome can be attributed to the increase in the number of molecules present in the solution.

Results for the polyglycine solutions are visualized in Figure 2, Panel G and H. The presence of polyglycine produced significant changes in absorption of the phosphatidylglycerol only solution. The increase in the area under the curve of the polyglycine solutions marks a decrease in the permeability of the liposomes.

The permeability of phosphatidyl glycerol with L-valglycylglycine

Fully formed vesicles were visualized under phase microscopy of phosphatidylglycerol combined with the tripeptide L-valglycylglycine. The goal of combining the tripeptide with the liposomes was to create a pore to allow increased permeability in the vesicles upon rehydration of the dried down solutes. Rehydrated solutions would hopefully not undergo extreme osmotic lysis due to the 10$100 x$ increase in the concentration of solute which occurs when dehydrating the liposomes. Each varying ratio did not change the number of vesicles present or the size of vesicles formed and did 
A)

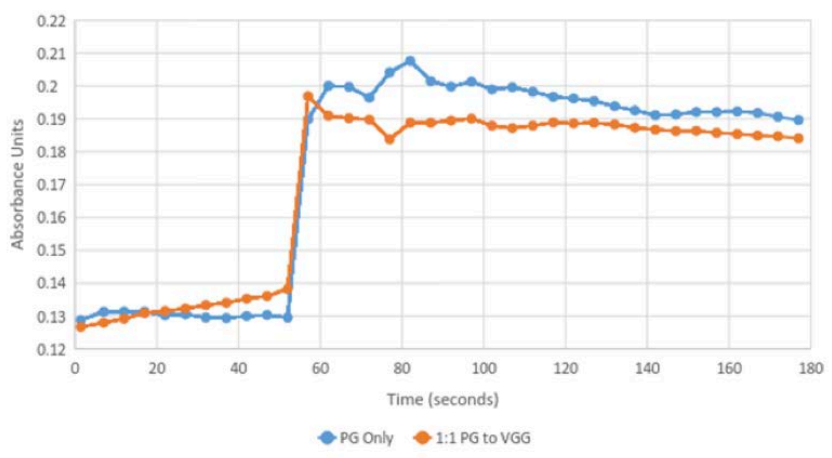

B)

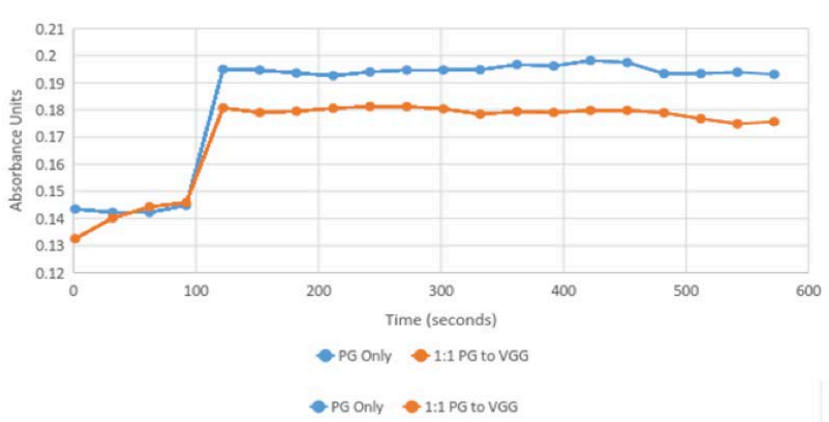

C)

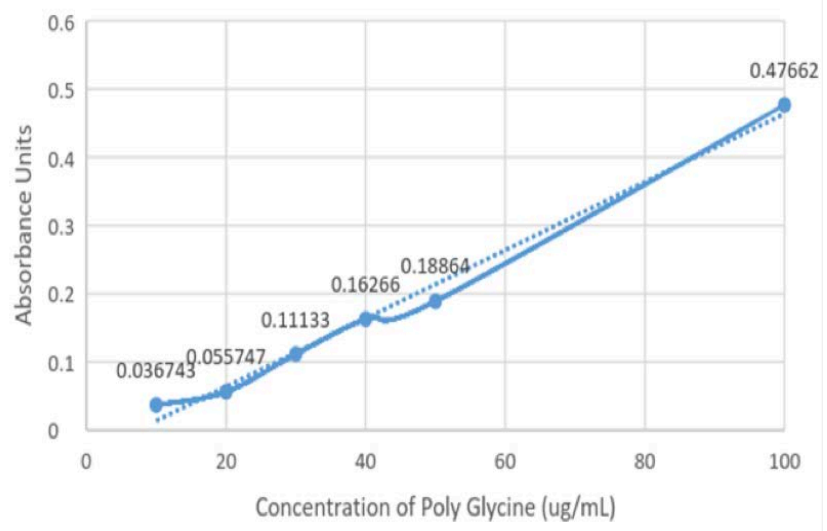

Figure 4. Spectrophotometry of phosphatidyl glycerol in solution with L-valglycylglycine Graph 10. A) Permeability of phosphatidyl glycerol with tripeptide pore formation. Test was done at 5-second intervals for 180 seconds. B) Permeability of phosphatidyl glycerol with tripeptide pore formation. Test was done at 30 -second intervals for 600 seconds. C) Standard curve of polyglycine at $200 \mathrm{~nm}$.

not produce any sort of inhibition or aggregation of the liposomes or amino acids. The results for L-valglycylglycine are visualized in Figure 3, Panels A-D.

Spectrophotometry of phosphatidyl glycerol in solution with L-valglycylglycine

Kinetic spectrophotometry of the tripeptide in phosphatidylglycerol shows that the permeability of the liposome did not vary from the permeability of the phosphatidylglycerol only solution. This confirms that the tripeptide failed to produce cross membrane pores within the vesicles. Spectrophotometry results and the standard curve for polyglycine is seen in Figure 4, Panels A-C.

Measuring absorbance of polyglycine bound to phosphatidyl glycerol

The absorbance and estimates of polyglycine bound to phosphatidylglycerol can be seen in Table 1. The amount of polyglycine bound to the liposomes per individual vesicle decreased as the ratio between phosphatidylglycerol and polyglycine increased. Quantifying the amount of polyglycine bound to the vesicles shows the $5: 1$ solution to have a $0.5: 1$ ratio of phosphatidylglycerol to bound polyglycine; the $10: 1$ solution to have a $1: 1$ ratio of phosphatidylglycerol to bound polyglycine; and the 20:1 solution to have a 2:1 ratio of phosphatidylglycerol to bound polyglycine. Binding to the liposomes occurs at about a 1/10 rate for each ratio.

Fluorescence addition to polyglycine and phosphatidyl glycerol

Fluorescent addition to polyglycine showed that binding did occur between the polypeptide residue and the lipid. The polyglycine is bound to the vesicle both outside and inside the phosphatidyl glycerol. Results can be seen in Figure 5.

Measuring the Osmotic Effects of Liposomes using Fluorescence

In solutions with polyglycine bound to labeled fluorescent phosphatidyl glycerol, polyglycine had no protective effects and microscopy showed fused vesicles and loss of fluorescence. With the addition of sucrose, and the osmotic effect was visualized with an observed loss of fluorescence, however, the vesicles took on a string-like form and were less fused than the polyglycine only solution. Results and observations can be seen in Table 2 .

\section{Microscopy of Fluorescence-Labeled Liposomes}

All solutions showed osmotic lysis of the liposomes and fusion of all vesicles, and thus, all fluorescence was lost, and the solutions were unable to be visualized with UV microscopy. Results are shown in Figure 6, Panels A-F. The phase microscopy shows fused vesicles much larger than the 1-micron vesicles which were extruded prior to dehydration.

Determining Lysis Prevention using polyglycine and polyglutamate

In order to determine if there is a general protecting effect from osmotic lysis from the addition of any molecule besides the lipid, we dehydrated and rehydrated solutions of polyglutamate and polyglycine present in vesicles that had been extruded to a 1-micron diameter. The polypeptides failed to provide protection from osmolysis, which would have been apparent by the inhibition of fusion of the vesicles. Results are shown in Figure 7, Panels A-E.

\section{DISCUSSION}

Our working hypothesis was to determine the potential of homopolymers as stabilization factors for simple lipid membranes. After combining homopolymers with liposomes and visualizing their interactions at varying ratios of lipid to polypeptide, we found substantial evidence that there were distinct conformational changes 


\begin{tabular}{ccc}
\hline $\begin{array}{c}\text { Ratio of phosphatidyl } \\
\text { glycerol to polyglycine }\end{array}$ & Absorbance & $\begin{array}{c}\text { Estimate of polyglycine } \\
\text { bound per vesicle }\end{array}$ \\
\hline $5: 1$ & 0.427 & $92.7 \mu \mathrm{g}$ \\
\hline $10: 1$ & 0.226 & $52.5 \mu \mathrm{g}$ \\
$20: 1$ & 0.078 & $22.9 \mu \mathrm{g}$ \\
\hline
\end{tabular}

Table 1. Measuring absorbance of polyglycine bound to phosphatidyl glycerol.

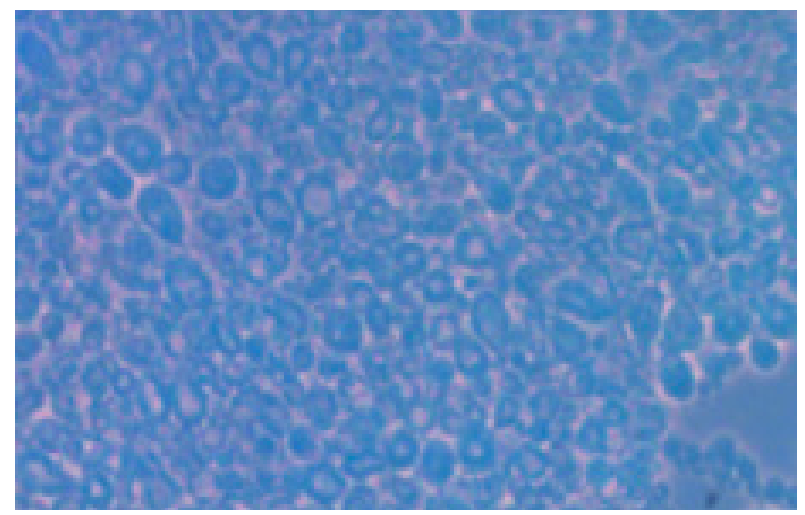

Figure 5. Fluorescamine-labeled polyglycine in a phosphatidyl glycerol solution under UV microscopy.

\begin{tabular}{ll}
\hline \multicolumn{1}{c}{ Solution } & \multicolumn{1}{c}{ Observations } \\
\hline $\begin{array}{l}\text { 5:1 polyglycine to labeled } \\
\text { phosphatidyl glycerol }\end{array}$ & $\begin{array}{l}\text { Polyglycine had no protective effect } \\
\text { on phosphatidyl glycerol. The micros- } \\
\text { copy of the solution showed fused ves- } \\
\text { icles and loss of phosphatidyl glycerol } \\
\text { fluorescence. }\end{array}$ \\
\hline $\begin{array}{l}\text { 10:1 polyglycine to labeled } \\
\text { phosphatidyl glycerol }\end{array}$ & $\begin{array}{l}\text { An osmotic effect was visualized } \\
\text { through loss of phosphatidyl glycerol }\end{array}$ \\
\hline $\begin{array}{l}\text { fluorescence. } \\
\text { phosphatidyl glycerol }\end{array}$ & $\begin{array}{l}\text { An osmotic effect was visualized } \\
\text { through loss of phosphatidyl glycerol }\end{array}$ \\
\hline $\begin{array}{l}\text { fluorescence. } \\
\text { phosphatidyl glycerol }\end{array}$ & $\begin{array}{l}\text { An osmotic effect was visualized } \\
\text { through loss of phosphatidyl glycerol } \\
\text { fluorescence. }\end{array}$ \\
\hline $\begin{array}{l}\text { Labeled phosphatidyl glyc- } \\
\text { erol with } 1 \text { mM sucrose }\end{array}$ & $\begin{array}{l}\text { Osmotic effect visualized and loss of } \\
\text { fluorescence observed. The vesicles } \\
\text { took on string-like form, and less fu- } \\
\text { sion of vesicles were observed. }\end{array}$ \\
\hline $\begin{array}{l}\text { Labeled phosphatidyl glyc- } \\
\text { erol with 100 mM sucrose }\end{array}$ & $\begin{array}{l}\text { Vesicles had all lysed, total fluores- } \\
\text { cence lost. Even less fusion than 1 mM } \\
\text { sucrose. }\end{array}$ \\
\hline $\begin{array}{l}\text { Unlabeled phosphatidyl glyc- } \\
\text { erol with } 100 \text { mM sucrose }\end{array}$ & $\begin{array}{l}\text { Same as labeled phosphatidyl glycerol } \\
\text { with } 100 \text { mM sucrose. }\end{array}$ \\
\hline
\end{tabular}

Table 2. Measuring the osmotic effects of liposomes using fluores-cence. in the liposomes upon interaction with varying charges of side chain residues. The strong ionic interaction of positively charged homopolymers with the negatively charged liposomal head groups suggested that high concentrations of positively charged homopolymers hinder the formation of membranes. And while polyglutamate did not produce inhibitive conformational changes like the positively charged residues, the side chains produced residues dissimilar to our control solution, whose vesicles were fully formed, spherical, and multilamellar, leading us to the conclusion that neither the positively nor negatively charged residues produce stabilizing effects. Polyglycine was the only compound whose interactions varied vesicle conformations was most comparable to the isolated lipid solution. These first preliminary results suggested to us that glycine may have the capacity to stabilize vesicles.

Kinetic spectrophotometry gave us another insight into the significance of homopolymer binding to vesicles in an osmotic environment. Despite increasing the absorbance and scattering effects of the solutions due to the increasing number of molecules present, neither the positively nor negatively charged peptides decreased the permeability of the membranes. Repulsive forces between the negatively charged carboxylate on the polyglutamate side chains could cause little interactivity between the liposomes and the protein, increasing the capacity for the solution to reach an equilibrium closer to the control absorption values. This corroborated prior evidence, indicating little protective effects from charged homopolymers. The presence of polyglycine extended peak absorbance by about 25 seconds. Hydrogen bonding interactions between the polar head group of phosphatidylglycerol and protons in the polyglycine residue may protect the integrity of the liposomes and make it more difficult for glycerol molecules to pass through to regain equilibrium, producing decreased permeability in a relatively osmotic environment. Through fluorescent visualization and quantitative confirmation of bound polyglycine residues to phosphatidyl glycerol, we can conclude that there were at least some minor stabilization effects of polyglycine for simple membranes.

Despite the presence of a small stabilizing effect of polyglycine, in highly osmotic environments we concluded that the osmotic effects produced upon rehydration of vesicles overwhelm any protective or stabilizing effects of polyglycine. Lost fluorescence in the polyglutamate solution suggested that polyglycine did, in fact, cause protective effects in permeability and that the type of molecules present in the solution has an influence on the permeability and protection of membranes. Our conclusion suggests that small protective effects from the hydrogen bonding interactions between the polar head groups of phosphatidylglycerol and the hydrogen residues on polyglycine account for the decreased permeability of the lipid membranes. These effects are ultimately not strong enough to protect from the osmotic effects which occur when rehydrating liposomes from multilamellar gel layers in the prebiotic environment. We could not rule out the potential of pores to provide enhanced stabilization, although due to shearing effects of pored vesicles it appears that there must be a primary stabili- 

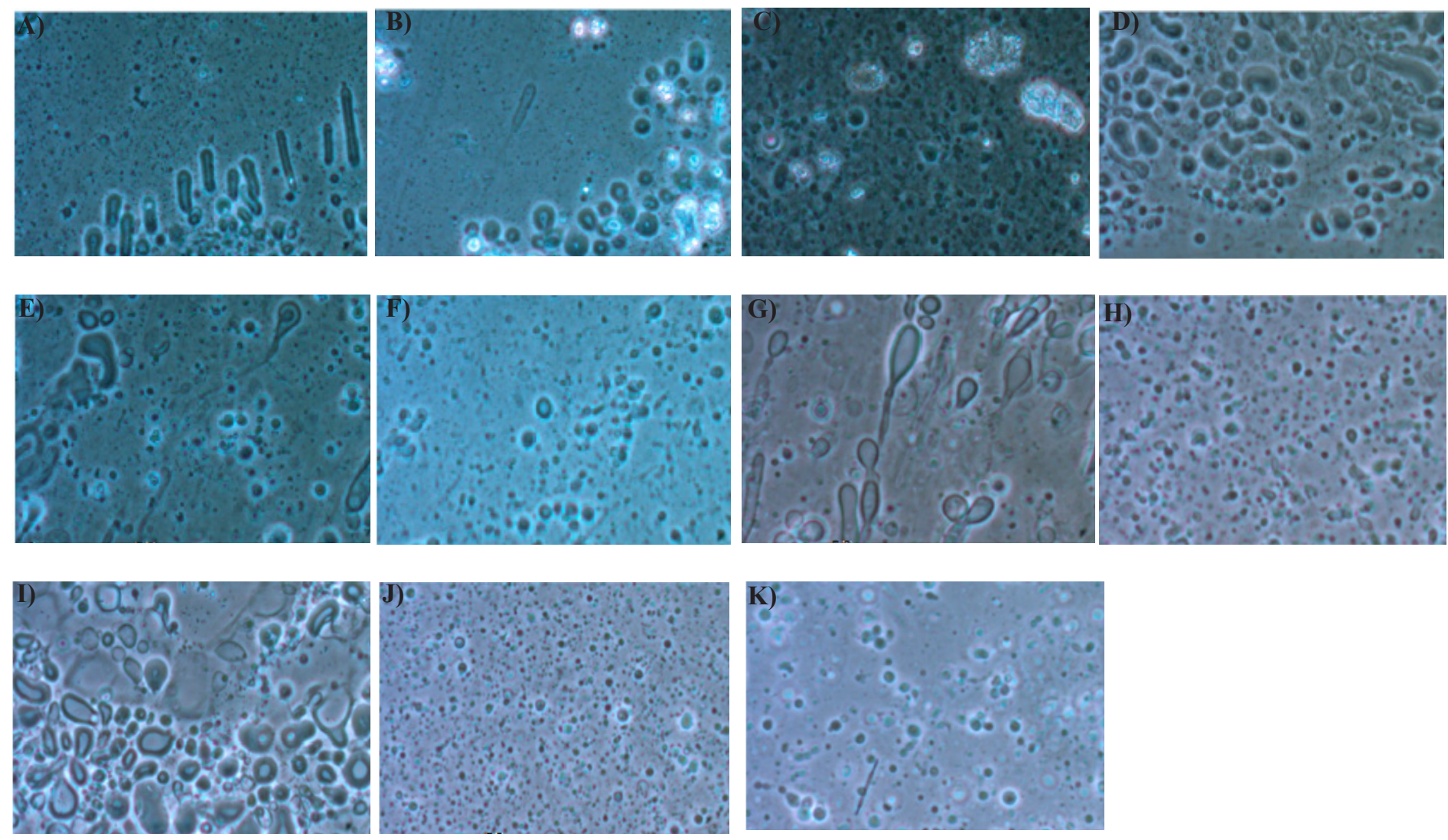

Figures 6A-F Microscopy of fluorescence-labeled liposomes. A) 5:1 polyglycine to labeled phosphatidyl glycerol. B) 10:1 polyglycine to labeled phosphatidyl glycerol. C) 10:1 polyglycine to unlabeled phosphatidyl glycerol. D) Labeled phosphatidyl glycerol in $1 \mathrm{mM}$ sucrose. E) Unlabeled phospha-tidyl glycerol in $1 \mathrm{mM}$ glycine. F) Unlabeled phosphatidyl glycerol in $100 \mathrm{mM}$ sucrose.

Figures 6G-K. Determination of osmotic lysis prevention. G) $0.5 \mathrm{~mL}$ phosphatidyl glycerol with $0.5 \mathrm{~mL}$ pyranine. H) $1: 1$ phosphatidyl glycerol to polyglycine $(0.5 \mathrm{mg} / \mathrm{mL}$ solution. I) $1: 1$ phosphatidyl glycerol to polyglycine $(2 \mathrm{mg} / \mathrm{mL})$ solution. J) $1: 1$ phosphatidyl glycerol to polyglutamate $(0.5 \mathrm{mg} / \mathrm{mL})$ solution. $\mathrm{K}) 1: 1$ phosphatidyl glycerol to polyglutamate $(2 \mathrm{mg} / \mathrm{mL})$ solution.

zation of the membranes prior to pore formation in the prebiotic environment.

Future steps to this research include creating pore structures in vesicles and adding polyglycine to determine if the addition of pores in conjunction with the protecting properties of glycine is sufficient to inhibit the lysis of prebiotic membranes during dehydration and rehydration cycles. Hydrative and dehydrative changes are a necessity to the synthesis of peptides in the prebiotic environment, however, osmolysis provides a huge potential problem for the hydrothermal hypothesis of the origin of life. Protecting factors would need to account for these osmotic effects and provide stabilization to the vesicles before and after hydration and dehydration cycles. Driving the reaction of monomeric components to synthesis is useless without the protection and compartmentalization of these products away from the harsh hydrothermal environments. Therefore, more experimentation is needed to explore the nature of osmotic effects on lipid membranes. Additionally, further illumination into the importance of temperature is needed to determine the potential of stabilizing membranes in the hydrothermal environment. The experiments conducted did not vary the temperature of solutions, which is a major force for driving synthesis forward in the prebiotic environment.

These experiments have increased our understanding of the interactions between amino acids and lipids in the hydrothermal environment, and this work can be used to understand basic thermodynamic principles of lipids in interaction with peptides, coming closer to answering the question of how newly synthesized residues are protected by liposomes in the prebiotic environment.

\section{ACKNOWLEDGMENTS}

Thank you to the University of California, Santa Cruz for allowing the advancement of scientific knowledge to move forward with the help of faculty and resources going towards undergraduate research. Thank you to my mentors who helped my studies along the way, and to Professor David Deamer for his guidance and counsel.

\section{REFERENCES}

Apel, C. L., Deamer, D.W, and Mautner, M.N. Self-assembled Vesicles of Monocarboxylic acids and Alcohols: Conditions for Stability and for the Encapsulation of Biopolymers. Biochimica Et Biophysica Acta. U.S. National Library of Medicine, 10 Feb. 2002. doi: 10.1016/S0005-2736(01)00400-X 
Cramer WA, Engelman DM, Von Heijne G, et al. : Forces involved in the assembly and stabilization of membrane proteins. FASEB J. 1992:3397-402

Damer, B., Deamer, D. Coupled Phases and Combinatorial Selection in Fluctuating Hydrothermal Pools: A Scenario to Guide Experimental Approaches to the Origin of Cellular Life. Life 2015, 5, 872-887. 13 March 2015. doi: 10.3390/ life 5010872

Fujioka, K., Futamura, Y., Shiohara, T., Hoshino, A., Kanaya, F., Manome, Y., and Yamamoto K.Amino acid Synthesis in a Supercritical Carbon Dioxide - Water System." International Journal of Molecular Sciences. Int J Mol Sci, 15 June 2009. doi: 10.3390/ijms 10062722.

Miller, S. A Production of Amino Acids Under Possible Primitive Earth Conditions. Science. American Association for the Advancement of Science, 15 May 1953.

Namani, T., and Deamer, D. W. Stability of model membranes in extreme environments. Origins of Life and Evolution of Biospheres, 38(4), 329-41. 2008.

Oliver A.E., Deamer, D. W. Alpha-helical hydrophobic polypeptides form protonselective channels in lipid bilayers.Biophysical Journal. 1994;66(5):13641379. 10.1016/S0006-3495(94)80927-0

Shimoyama, A., Namaoka, H., Komiya, M., Hanada, K. (199) Analyses of carboxylic acids and hydrocarbons in Antarctic carbonaceous chondrites. Geo chem J 23:181-193. 11 Dec. 1989 